\title{
Uterine natural killer cells: insights into their cellular and molecular biology from mouse modelling
}

B. Anne Croy ${ }^{1}$, Hong He ${ }^{1}$, Souad Esadeg ${ }^{1}$, Qingxia Wei ${ }^{2}$, Daniel McCartney ${ }^{1}$, Jianhong Zhang ${ }^{1}$, Angela Borzychowski ${ }^{1}$, Ali A. Ashkar ${ }^{3}$, Gordon P. Black ${ }^{1}$, Sharon S. Evans ${ }^{4}$, Sirirak Chantakru ${ }^{5}$, Marianne van den Heuvel ${ }^{1}$, Valdemar A. Paffaro, $\mathrm{Jr}^{6}$ and Aureo T. Yamada ${ }^{6}$

${ }^{1}$ Department of Biomedical Sciences, Ontario Veterinary College, University of Guelph, Guelph, ON, N1G 2W1, Canada; ${ }^{2}$ Arthur and Sonia Labatt Brain Tumor Research Centre, Hospital for Sick Children, 555 University Avenue, Toronto, ON, M5G 1X8, Canada; ${ }^{3}$ Department of Pathology and Molecular Therapeutics, McMaster University, 1200 Main Street W., Hamilton, ON, L8N 3Z5, Canada;

${ }^{4}$ Department of Immunology, Roswell Park Cancer Institute, Elm at Carlton Streets, Buffalo, NY 14623, USA; ${ }^{5}$ Department of Anatomy, Kasetsart University, 50 Pahonyothin Road, Chatucak BangKhen, Bangkok, 10900, Thailand; and ${ }^{6}$ Department of Histology and Embryology, Institute for Biology, UNICAMP, Campinas, 13083-970, S.P. Brazil

In primates, including women, and in rodents, natural killer lymphocytes (NK cells) have a unique relationship with the decidualizing uterus. Implantation sites from genetically modified and transplanted mice have proven useful models for understanding potential mechanisms involved in the recruitment, activation and functions of human CD56 bright uterine $(\mathrm{u}) \mathrm{NK}$ cells. Key findings are reviewed in this article. In mice, uNK precursor cells are recruited from secondary lymphoid tissues and are activated coincident with their uterine arrival. uNK cells proliferate, produce cytokines (interferon gamma (IFN- $\gamma$ ) and interleukin 18 (IL-18) and IL-27), and terminally differentiate into granulated lymphocytes. Many uNK cells proliferate within the myometrium at each implantation site forming a structure, the mesometrial lymphoid aggregate of pregnancy (MLAp) that surrounds blood vessels servicing each placenta. Post-mitotic uNK cells are abundant within decidua basalis; frequently ( $>25 \%$ ) associating with spiral arteries, intramurally and intraluminally. From mid-gestation, numbers of uNK cells decline. Studies of implantation sites in mice lacking uNK cells, IFN- $\gamma$, components of IFN- $\gamma$-induction and -signalling pathways or IFN- $\gamma$-regulated genes indicate that $\mathrm{uNK}$ cell-derived IFN- $\boldsymbol{\gamma}$ is essential in triggering pregnancy-induced spiral artery modification. Decidual maintenance and uNK cell death are additional effects of uNK cell-derived IFN- $\gamma$. Thus, during the first half of gestation, uNK cells contribute to and sustain important changes in the maternal placental bed.

During uterine decidualization in women, non-human primates and rodents, lymphocytes of the natural killer (NK) lineage appear and become abundant as large, granulated cells (Fig. 1; Parr et al., 1987; Peel, 1989; Moffett-King, 2002). NK cells differ from T and B lymphocytes because they lack somatically rearranged antigen-sensing receptors (Natarajan et al., 2002). NK

Email: acroy@uoguelph.ca cells contribute to innate immunity, participating in early immune protection, before clonal expansion of $\mathrm{B}$ and $\mathrm{T}$ lymphocytes. Functions of NK cells are lysis and cytokine production, with individual cells having single or dual capacity. Lysis is directed against virally infected cells and tumour cells. Interferon-gamma (IFN- $\gamma$ ), which restricts viral infection, is a major cytokine product (Trinchieri, 1995). Uterine (u)NK cells are predominantly activated, cytokine-producing NK cells (Moffett-King, 2002). Fifteen years lapsed between NK cell identification in marrow, blood and spleen and recognition of endometrial granulocytes (human designation) and 


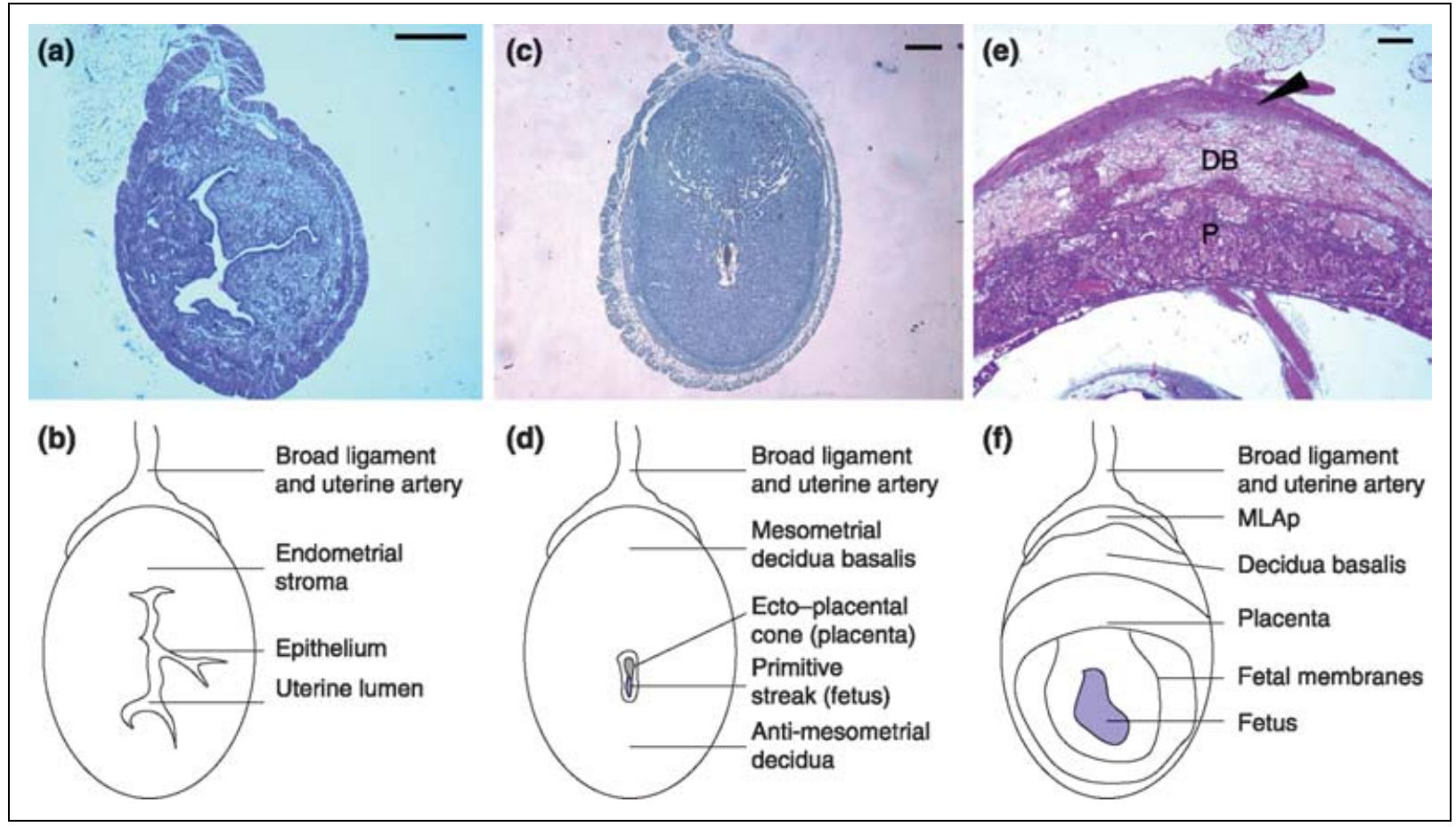

Fig. 1. (a,c,e) Photomicrographs and $(b, d, f)$ line drawings of the $(a, b)$ virgin, $(c, d)$ day 6 of gestation and $(e, f)$ day 10 of gestation mouse uteri. In all images, the uterine artery access through the broad ligament (mesometrium) is at the top. Implantation triggers transformation of endometrial stroma into decidua. This process begins anti-mesometrially and progresses mesometrially (compare (a) with (c)), resulting in formation of the decidua basalis (DB) and loss of the uterine lumen. Uterine natural killer (uNK) cells are first found in the DB. As additional structures differentiate, uNK cells also localize between the layers of the uterine wall, in a transient structure called the mesometrial lymphoid aggregate of pregnancy (MLAp), marked with an arrowhead in (e), and are found at lower frequency within the placenta (P). (a) and (e) Stained with haematoxylin and eosin; (c) stained with the lectin Dolichos biflorus agglutinin (DBA) and haematoxylin. uNK cell granules are distinctive in sections stained with periodic acid-Schiff (PAS) reagent or DBA lectin (Peel, 1989; Paffaro et al., 2003). DBA lectin also stains uNK cell plasma membranes but PAS does not (Paffaro et al., 2003). uNK cells are somewhat difficult to recognize by haematoxylin and eosin staining as their granules, which are histochemically eosinophilic, are relatively indistinct. Virgin uterus is DBA lectin-negative. Scale bars represent (a) $400 \mu \mathrm{m}$ and (c,e) $200 \mu \mathrm{m}$.

granulated metrial gland cells (murine designation) as NK cells.

uNK cells share many, but not all, of their features with peripheral NK cells. For example in humans, most blood NK cells analysed by flow cytometry express the surface marker CD16, an immunoglobulin domain receptor, and have dim expression of CD56, an adhesion molecule. About $1 \%$ of circulating lymphocytes are $\mathrm{CD}^{-} 6^{-}$, CD56 bright $\mathrm{NK}$ cells and these co-express high amounts of the vascular addressin L-selectin (Campbell et al., 2001). In contrast, most human uNK cells express CD56 brightly but lack CD16 and L-selectin (Searle et al., 1999). Lymphotoxin $\beta$ receptor (LT $\beta R$ ) is a murine example. LT $\beta R$ is essential for peripheral NK cell differentiation and secondary lymphoid tissue formation (Fu and Chaplin, 1999). However, uNK cells differentiate and an architecturally correct mesometrial lymphoid aggregate of pregnancy (MLAp) forms in pregnant $\mathrm{LT}^{\circ} \% \mathrm{R}^{\circ}$ mice (Kather et al., 2003). Such findings limit the usefulness of studying NK cells not isolated from or functioning within the uterus and make the unique features of
uNK cell recruitment, activation and differentiation key questions. It is of critical importance to recognize that these processes occur without pregnancy or fetal trophoblast tissue. In women, uNK cells differentiate in every menstrual cycle, 3-5 days after the LH surge (Bulmer et al., 1987; King, 2000). In rodents, induction of artificial deciduomata induces fully mature uNK cells (Peel, 1989).

The question of uNK cell function during pregnancy is also of tremendous interest. The cells are highly mobile and their high content of lytic molecules plus partnering antigen receptor display makes them potentially dangerous to trophoblast cells in implanting primate blastocysts and in developing placentae (primates and rodents). uNK cells could play essential physiological roles limiting normal trophoblast invasion or could destroy trophoblast, leading to pregnancy loss. Full definition of uNK cell antigen recognition on trophoblast and the intracellular signalling involved in uNK cell activation, differentiation and senescence would resolve such functional debates and provide clinically relevant 
Table 1. Summary of histological findings in uterine natural killer (uNK) cell-deficient mice with or without bone marrow transplantation

\begin{tabular}{|c|c|c|c|c|c|c|}
\hline \multicolumn{2}{|c|}{ Strain } & \multirow[b]{2}{*}{ Manipulations } & \multicolumn{4}{|c|}{ Histological findings } \\
\hline Phenotype & Name & & uNK & DB & SA & MLAp \\
\hline Normal & $\begin{array}{l}\text { C57Bl/6J } \\
129 \mathrm{~J} \\
\text { Balb/cJ } \\
\text { CD1 }\end{array}$ & - & + & Cellular & Modified & Present \\
\hline $\mathrm{T} \pm \mathrm{B}$ deficient & $\begin{array}{l}\text { Nude } \\
\text { SCID }\end{array}$ & - & + & Cellular & Modified & Present \\
\hline $\begin{array}{l}\text { NK/uNK, } \\
T \pm B \text { deficient }\end{array}$ & $\begin{array}{l}\text { IL-2/15R } \beta^{\circ /} \\
\text { IL-2/15R } \gamma^{\circ}(\gamma \mathrm{C}) \\
\mathrm{IL}-15^{\circ} \% \\
\mathrm{Tg} \in 26 \\
\mathrm{RAG}-2^{\circ} / \gamma \mathrm{C}^{\circ}\end{array}$ & - & - & Hypocellular & Unmodified & Absent \\
\hline $\begin{array}{l}\text { Bone marrow graft } \\
\text { into uNK deficient }\end{array}$ & RAG- $2^{\circ} / \gamma \mathrm{C}^{\circ} \%$ & $\begin{array}{l}+ \text { SCID BM } \\
+ \text { C57BI/6J BM } \\
+ \text { IL-15\% BM } \\
+\beta E R K O B M \\
+\alpha \text { ERKO BM }\end{array}$ & + & Cellular & Modified & Present \\
\hline
\end{tabular}

BM: bone marrow; DB: decidua basalis; ERKO: oestrogen receptor knockout mouse; MLAp: mesometrial lymphoid aggregate of mouse pregnancy; RAG- $2^{\circ / \gamma} \mathrm{c}^{\circ / \%}$ : double knockout mouse for recombinase activating gene-2/common cytokine chain gamma; SA: spiral artery of the decidua basalis; SCID: severe combined immunodeficient.

information. Mice have proven excellent models for characterization of uNK cell biology by allowing manipulative studies that are not possible in pregnant women (Table 1 ). Strains of genetically mutant mice are available to address uNK cell regulation and studies are relatively short in duration (mouse pregnancy is 19-20 days). Furthermore, murine pregnancies can be interrupted and entire implantation sites studied. Inbred strains permit investigations of replicate pregnancies in time-course analyses that precisely define the changing cell and tissue relationships during gestation (Fig. 1). Murine uNK cell analyses are usually histological or molecular because no successful culture conditions or long-term cell lines are known. In the present study, at least six implantation sites derived from two or three pregnant females on at least two different days of gestation were serially sectioned. From each implant site eleven central sections were selected for computerbased image analysis in a manner ensuring that no duplicate counting of individual cells occurred. Control pregnancies were matched by genetic background as closely as possible, being sibling matings or matings of a congenic inbred partner strain matched for day of gestation. Study of human uNK cells occurs at more restricted times, largely those associated with biopsy or elective termination, and is genetically heterogeneous. In addition to histopathology, flow cytometry is widely used to study lymphocytes dissociated from human specimens and primary cloning has been achieved (Christmas et al., 1990). Application of expression microarrays and laser capture microdissection combined with quantitative analyses will provide information unique to human uNK cells and their environment, an environment distinct from that of the rodent anatomically, endocrinologically and in duration of gestation. The mouse will remain a key tool in critical assessment of new data from women because this model can move studies from correlative findings to genetically defined in vivo gestational data with potential for experimental interventions. UNK cells are infrequent or absent at term in both mice and women (Delgado et al., 1996; King, 2000), making term tissue irrelevant for defining uNK cell functions.

\section{NK cell differentiation and establishing the uNK cell lineage}

\section{Origins}

NK cells are generated from pluripotent bone marrow stem cells. Their differentiation in mice is highly dependent upon stromal factors including stem cell factor, interleukin 7 (IL-7) and IL-15 (Kennedy et al., 2000; Rosmaraki et al., 2001). Lineage-committed NKprecursor cells have limited self-renewal ability and are present in secondary lymphoid tissues (blood, spleen, lymph nodes (LN)) but not the uterus (Chantakru et al., 2002 and references therein). Transplants from NK cellsufficient mice to NK cell-deficient mice established that UNK precursors are present in fetal liver; fetal, neonatal and adult thymus; marrow, LN and spleen (Chantakru et al., 2002). Only spleen showed enhanced ability to generate uNK cells if harvested from pregnant donors (days 3-7 of gestation), whereas LN draining 


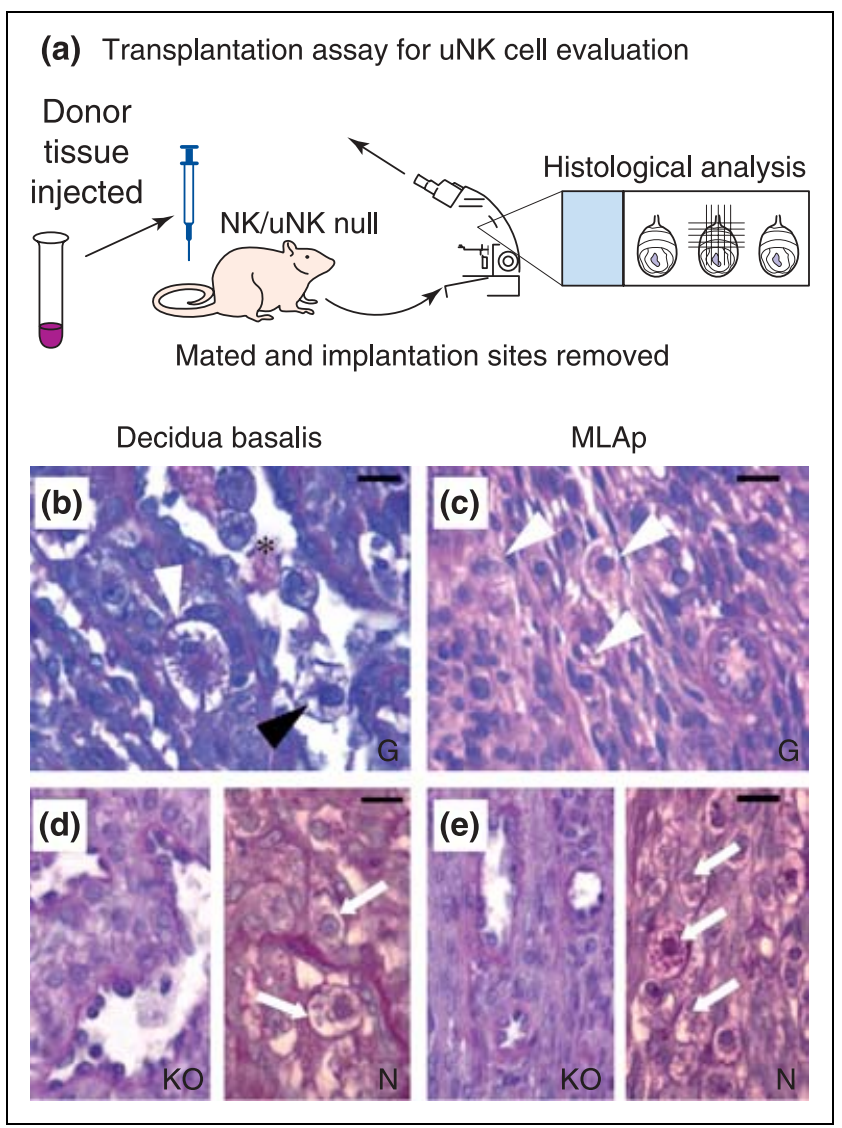

Fig. 2. The in vivo assay system, used to assess uterine natural killer (uNK) cell precursor differentiation, homing and regulation, and to define uNK cell functions, depends on pregnancy in mice genetically deficient in the NK/uNK cell lineages. At present, the most reliable mouse used for these studies and manipulations is the RAG- $2 \% / \gamma \mathrm{C}^{\circ} / \%$ double knockout. The RAG- $2^{\%} / \gamma \mathrm{c}^{\circ}$ is alymphoid but fertile under appropriate housing. Cell suspensions or tissues are grafted into virgin or mated uNK cell-deficient recipients. Donors can be normal or altered in the gene(s) of interest. At different times after transplantation, implantation sites are analysed for graft-derived uNK cells. Any and all uNK cells will be graftderived in RAG- $2^{\circ / \gamma} / \mathrm{c}^{\circ}$ recipients. Independent microdomains (usually the decidua basalis (DB) and the mural, mesometrial lymphoid aggregate of pregnancy (MLAp)) are assessed within each implantation site. The procedure is summarized in (a). (b,c) Graft-derived uNK cells in the DB and MLAp (white and black arrowheads, respectively), 10 days after inoculation of peripheral lymph node cell suspensions from $\mathrm{C} 57 \mathrm{BI} / 6 \mathrm{~J}$ mice at day 5 of gestation to mated RAG- $2^{\circ} / \mathrm{yc}^{\mathrm{o}} / \mathrm{r}$ recipients at day 0 of gestation. uNK cells in the MLAp are smaller, have fewer cytoplasmic granules and more mitotic figures than those in the DB (compare (c) with (b)), findings replicating implantation sites in pregnant, genetically normal mice. (b) The common intravascular $(*)$ position of uNK cells (black arrowhead) in the DB is replicated in transplant recipients. (d) The DB in unmanipulated RAG- $\left.2 \% / \gamma \mathrm{c}^{\mathrm{o}} / \mathrm{KO}\right)$ and C57Bl/6J (N) females at day 10 of gestation. (e) The MLAp in unmanipulated RAG- $2 \% / \gamma \mathrm{C}^{\%}(\mathrm{KO})$ and $\mathrm{C} 57 \mathrm{BI} / 6 \mathrm{~J}(\mathrm{~N})$ females at day 10 of gestation. No uNK cells are found in unmanipulated RAG $-2 \% / \gamma \mathrm{C}^{\circ} \%$ but numerous uNK cells (arrows) are present in these tissues in normal mice. Scale bars represent $20 \mu \mathrm{m}$; all sections are stained with periodic acid-Schiff. pregnant uteri were devoid of uNK precursors (Fig. 2). These observations strongly indicate that, before implantation, endocrine signals co-ordinately mobilize uNK precursors from spleen and induce mechanisms for trapping and retaining these circulating cells within the uterus (Chantakru et al., 2002).

\section{Ovarian hormone effects}

The present authors addressed functionally the postulated steroid hormone-regulated interactions between lymphocytes and endothelium by assaying human blood lymphocyte adhesion to high endothelial venules in cryostat sections of mouse subcutaneous LN pools (PLN). Lymphocytes from anonymous blood-bank donors were evaluated using PLN from virgin and pregnant mice. Endothelium from pregnant donors attracted more cells (Chantakru et al., 2002). Endothelium in PLN from ovariectomized mice given oestradiol or progesterone or both also gained adhesive function equalling pregnancyinduced gains. Antibody-blocking studies established that L-selectin and $\alpha 4$-integrin ligands mediated the functional changes. Adhesion assays were extended using mouse uterine tissues as substrate (virgin, pregnant or steroid hormone-treated) instead of PLN. Binding again occurred via L-selectin and $\alpha 4$-integrin-dependent mechanisms and numbers of cells adhering per $\mathrm{mm}^{2}$ of uterus were increased if tissue came from pregnant or steroid hormone-treated (oestrogen, progesterone, or both) ovariectomized mice. Prelabelling the human lymphocytes with anti-CD56 revealed that gains in functional adhesion to decidua basalis (DB) occurred in both $\mathrm{CD}^{2} 6^{+}$and $\mathrm{CD}^{-} 6^{-}$cells but greatest enrichment occurred in $\mathrm{CD}_{56} 6^{+}$cells ( $>70$-fold compared with the starting blood-cell suspension; Chantakru et al., 2002). Gain in ability of endothelium to attract lymphocytes was not systemic, as no pregnancy- or steroid hormoneinduced changes were observed in pancreatic endothelium (Chantakru, 2002; S. Chantakru, W-C Wang, B. A. Croy and S. S. Evans, unpublished).

Two studies were performed to determine whether hormones regulate lymphocytes independently but coordinately with endothelium. First, splenic lymphocyte suspensions from virgin, pregnant or ovariectomized mice treated with placebo, or oestrogen in the presence or absence of progesterone, were tested for adhesion to PLN from a single donor. Adhesion rates for splenocytes from virgin and ovariectomized, placebo-treated mice were similar and statistically lower than adhesion of splenocytes from pregnant or steroid hormone-treated donors, indicating that pregnancy, oestrogen and progesterone enhance the ability of murine lymphocytes to interact with endothelium (Chantakru, 2002; S. Chantakru, W-C Wang, B. A. Croy and S. S. Evans, unpublished). For the second study, blood samples were collected from women three times per week over their 
menstrual cycle and adhesion to constant mouse tissue was assessed. A dynamic, predictable pattern was found with significant gains in cells adhering to PLN and to decidualized uterus at the LH surge (M. van den Heuvel, S. Bashar, J. E. Lewis, X. Xie, J. Horrocks and B. A. Croy, unpublished). These data are consistent with a hypothesis of periovulatory mobilization of human uNK cell precursors to blood and their uterine appearance at LH+3-5 (King, 2000). Limited duration and importance of the luteal phase in mice may explain why different hormones appear to mediate the pregnancy-associated gains in lymphocyte-endothelial interactions in women and mice. Whether these dynamic changes reflect important steps in vivo in recruitment of uNK precursors to human uteri remains to be addressed experimentally. In an in vivo model of fever-range hyperthermia, doubling in vitro adhesion correlated with quadrupled lymphocyte trafficking to the targeted site (Evans et al., 2001).

\section{IL-15}

IL-15, a stromal cell and macrophage-derived cytokine, is critical for NK cell differentiation in human and murine lymphoid tissue (Carson et al., 1994; Kennedy et al., 2000). IL-15 acts on precursor and immature NK cells but not during NK progenitor cell differentiation. Human and murine endometria express IL-15 (Ye et al., 1996; Kitaya et al., 2000; Okada et al., 2000; Dunn et al., 2002). In mice, IL-15 mRNA is transcribed between day 6 and day 11 of gestation (Ye et al., 1996; A. A. Ashkar, G. P. Black, Q. Wei, J. R. Head and B. A. Croy, unpublished). In humans, IL-15 transcription is more abundant during the secretory than during the proliferative cycle phase and is sustained in early pregnancy with localization to endothelium and perivascular stromal cells of decidual spiral arteries (SA; Kitaya et al., 2000). These correlative time-course data strongly indicate that IL-15 participates in UNK cell differentiation, whereas the localization data indicate that IL-15 may contribute to chemotactic localization of uNK cells within the lumina and walls of the spiral arteries.

IL-15 shares two of its three receptor chains ( $\beta$ and $\gamma$ ) with IL-2, a cytokine not normally found in gestational uteri. Mice genetically ablated for either shared receptor or for IL-15 do not differentiate uNK cells (Guimond et al., 1998; Croy et al., 2003). Reconstitution and blocking bone marrow transplantation experiments were conducted to establish that IL-15 contributes to terminal $\mathrm{uNK}$ cell differentiation. The reconstitution experiment involved transplants between two strains that do not differentiate uNK cells. Alymphoid mice (RAG-2\%/common cytokine receptor chain $\gamma(\gamma \mathrm{c})^{\circ}$, a double knockout strain that makes IL-15) were grafted with bone marrow from IL-15\% mice, then mated and studied histologically. uNK cell differentiation occurred at frequencies identical to those observed in normal congenic $\mathrm{C} 57 \mathrm{Bl} / 6 \mathrm{~J}$ mice. For the blocking experiment, $\mathrm{C} 57 \mathrm{Bl} / 6 \mathrm{~J}$ bone marrow was given to IL-15\% mice. This bone marrow produces normal uNK cells when grafted into alymphoid mice (Ashkar et al., 2000), but was totally blocked from differentiation in uteri of IL-15\% recipients (Croy et al., 2003; A. A. Ashkar, G. P. Black, Q. Wei, J. R. Head and B. A. Croy, unpublished). These experiments indicate that IL-15 is the crucial factor regulating uNK cell differentiation and provoke questions concerning the regulation of uterine IL-15 and whether its deficiency or over-abundance has clinical consequences in women, as suggested by Chegini et al. (2002) for women who experience recurrent spontaneous abortions. Interferon regulatory factor (IRF)-1, a key regulator of IL-15 in marrow, is expressed in the uterus in humans and mice (Jabbour et al., 1999; Kitaya et al., 2001; A. A. Ashkar, G. P. Black, Q. Wei, J. R. Head and B. A. Croy, unpublished) but does not appear to regulate murine uterine IL-15 (Ashkar and Croy, 1999; A. A. Ashkar, G. P. Black, Q. Wei, J. R. Head and B. A. Croy, unpublished). In humans, uterine IRF-1 appears to be regulated by prolactin (Jabbour et al., 1999), whereas uterine IL-15 expression appears to be regulated by progesterone (Okada et al., 2000; Kitaya et al., 2000) and prostaglandins (Dunn et al., 2002). Additional information on uterine specific regulation of IL-15 is needed.

Clearly, ovarian steroid hormones act on uterine stroma, including endothelium, as discussed above, in ways that promote differentiation of uNK cells, but whether the hormones act directly on the lymphocytes or their precursors is less clear. Availability of mice ablated for the oestrogen receptor (ER) $\alpha$ or $\beta$ ( $\alpha$ ERKO and $\beta E R K O)$ (Couse and Korach, 1999) permitted functional assessment of ER utilization by uNK cells. Marrow transplanted from $\alpha E R K O$ and $\beta E R K O$ to alymphoid mice fully reconstituted uNK cells, indicating no direct functional action in mouse uNK cells through oestrogen receptors. This conclusion was supported by RT-PCR analysis of highly purified (99\%) uNK cells at days 12 and 13 of gestation, isolated rapidly from perfused, normal (C57Bl/6J) mice using a newly reported protocol involving magnetic beads coated with the lectin Dolichos biflorus agglutinin (DBA) (Borzychowski et al., 2003; Paffaro et al., 2003). mRNA for ER $\alpha$ and $E R \beta$ were absent, whereas mRNA for other genes was detected (Borzychowski et al., 2003). Human uNK cells have recently been reported to be $E R \alpha$ negative, as in earlier studies, but to express ER $\beta$ (Henderson et al., 2003), indicating species difference or transcripts from rare contaminating cells. In both humans and mice, studies of the complex interactions between the endocrine and stromal environments on lymphocyte differentiation within the uterus will be profitable and of clinical importance for endometrial as well as reproductive health. 


\section{NK cell and uNK cell activation}

Lymphocyte activation requires signals additional to those involved in lymphocyte differentiation. IL-12 is a cytokine important for induction of IFN- $\gamma$ synthesis in NK and T cells (Trinchieri, 1995). The action of IL-12 is enhanced by IL-18 (Nakanishi et al., 2001). IL-12 and IL-18 are found in normal human and mouse implantation sites (Devergne et al., 2001; Yoshino et al., 2001; Zourbas et al., 2001; Chaouat et al., 2002; Zhang et al., 2003). IL-18 shows a dynamic protein expression pattern by immunohistology, appearing first in decidual stromal cells at days 4-6 of gestation, then exclusively from day 8 to day 14 of gestation in uNK cells (Chaouat et al., 2002; Zhang et al., 2003). Pregnant mice deficient in both IL-12 and IL-18 (Takeda et al., 1998) have been compared with littermates deficient in only one or neither cytokine (Zhang et al., 2003). Females and males matched in genotype were mated. uNK cell differentiation was morphologically and numerically similar in the four strains. In the three cytokine-deficient strains, mid-gestation IFN- $\gamma$ (after day 8 of gestation) was somewhat reduced and $\mathrm{SA}$ dilation was impaired but not as severely as in IFN- $\boldsymbol{\gamma}^{\circ /}$ implantation sites. This finding indicated that additional cytokines contribute to UNK cell activation. Two recently described cytokines, IL-23 and IL-27, are related to IL-12 and contribute to IFN- $\gamma$ induction (Parham et al., 2002; Pflanz et al., 2002 and references therein). IL-23 and IL-27 expression is absent from virgin mesometrial uterus and mesometrial uterus at day 3 of gestation, but present in mesometrial decidua from day 4 of gestation (Zhang et al., 2003). Analyses of mRNA, from DBA lectin-purified uNK cells, indicate that stromal cells produce IL-23, whereas UNK cells produce IL-27. The time-course patterns for induction of these cytokines match well with putative roles for these cytokines in uterine IFN- $\gamma$ regulation in normal mice because IFN- $\gamma$ protein is absent mesometrially from virgin and pre-implantation uteri at day 3 of gestation but is detected from day 6 of gestation by ELISA. Peak mesometrial IFN- $\gamma$ occurs at day 10 of gestation and then declines (Ashkar et al., 2000).

NK cells interact with other cells via surface receptors that are classified as activating or inhibitory, depending on whether lytic activity is displayed when the receptor is engaged in in vitro assays. Individual cells display multiple receptors to give target recognition specificity. The ligands initially defined for NK cell receptors were classic major histocompatability complex (MHC) class I molecules or their structural relatives (Cerwenka and Lanier, 2001; Natarajan et al., 2002). Because the pathway resulting in terminal uNK cell differentiation in primates and rodents is triggered in the absence of conception, the role of non-self antigen recognition during uNK cell activation is unclear. Trophoblast has restricted transplantation antigen expression that, in outbred matings, will include paternally derived, non-self antigens (Moffett-King, 2002). The human uNK cell population expresses a full repertoire of immunoglobulinlike NK cell receptors, although in different proportions than expressed by blood NK cells (Hiby et al., 1997). The major NK cell receptors in mice are lectin-like LY49 gene family members. uNK cells from C57Bl/6 mice express all of the LY49 NK receptors typical of the strain (V. A. Paffaro, Jr, H. He and B. A. Croy, unpublished). This sharing of receptors between blood NK and UNK cells indicates that uNK cell activation will display many features in common with NK cell activation in other tissues and predicts that the unique properties of uNK cells are not defined by these receptors.

A second type of NK cell activation receptor in mice and humans is NKG2D. The Rae- 1 family of five retinoic acid-induced oncodevelopmental antigens was recently found to engage murine NKG2D, whereas the homologous human ligands are products of the HLAassociated genes MICA and MICB (Cerwenka and Lanier, 2001). Interactions between murine NK cells and Rae-1 genes are normally studied in vitro using embryonic cellline targets. In vivo expression of Rae-1 is reported only in mid-gestation developing mouse brain (Nomura et al., 1996). The present authors addressed transcription of Rae $1-\alpha-\beta-\gamma-\delta-\epsilon-$ in uteri from $C 57 \mathrm{Bl} / 6$ and $129 / \mathrm{J}$ strains by RT-PCR. Non-pregnant uteri expressed no Rae-1 signal but strain appropriate Rae- 1 expression was induced by day 6 of gestation in DB and sustained at day 10 of gestation in DB, MLAp and placenta. Immunohistochemistry using a pan Rae- $1 \alpha$ polyclonal antibody showed that extra-fetal expression was most strongly localized to trophoblast (B. A. Croy and T. Seya, unpublished). These and other developmental antigens expressed by trophoblast may be of major importance in sustaining uNK cell activation when trophoblasts and NK cells begin to intermingle (in mice from about day 8 of gestation). Roles for oncodevelopmental antigens are not yet explored in humans.

\section{Functions of uNK cells}

$u N K$-deficient and IFN- $\gamma$ signal disrupted mice

Several mouse strains have major (>99\%) or absolute deficits in NK cells. Five strains, each having different gene deletions, are uNK cell-deficient and have common histopathology (Table 1; Guimond et al., 1998; Ashkar et al., 2000). Three anomalies accompany absence of uNK cells: absence of myometrial MLAp development (that is, a decidual bed deficit), hypocellularity and oedema of DB and persistence of vascular smooth muscle in the SA with limited lumen dilation and vessel lengthening at mid-gestation. There is no overgrowth of trophoblast and no consistent quantifiable impairment in fetal or postnatal health (Greenwood et al., 2000). Issues such as maternal vascular or fetal gene expression, fetal hypoxia, brain development, ventricular hypertrophy, 
maturity onset diabetes and others have not been addressed experimentally between uNK-deficient and uNK cell-sufficient gestations. These findings imply that the normal functions of uNK cells during pregnancy are to congregate lymphocytes myometrially at the portals of uterine arteries and veins, to interact with and provide growth support for stromal cells committed to decidual differentiation, and to trigger events that culminate in normal, pregnancy-induced SA modification. These interpretations were confirmed by normalizing implantation sites in NK/uNK cell-deficient mice by bone marrow transplants from severe combined immunodeficient (SCID) (T-B-) mice (Guimond et al., 1998). Effector mechanisms were addressed by transplanting NK/uNK celldeficient females with bone marrow deleted for IFN- $\gamma$, IFN- $\gamma \mathrm{R} \alpha$ or their downstream signalling molecule Stat- 1 . Implantation sites in the recipients revealed that IFN- $\gamma$ is not required to initiate UNK cell differentiation but low concentrations are required for full maturation and senescence of uNK cells. Terminal uNK cell maturation required about 1 iu per implantation site, which is adequately provided by other types of cell (Ashkar et al., 2000). uNK cell-derived IFN- $\gamma$ provides higher tissue contents (>6 iu per implantation site) and these contents support decidual integrity and SA modification. Daily infusions of mrIFN- $\gamma$ (100-3000 iu per treatment) into alymphoid females at days $6-11$ of gestation were effective in induction of normal SA modification and decidual maintenance in the absence of uNK cells (Ashkar et al., 2000). Thus, pro-inflammatory cytokines act physiologically in normal pregnancy.

The $\alpha_{2}$-macroglobulin gene family, possible targets of $u N K$-cell derived IFN- $\gamma$

As the present study indicated that IFN- $\gamma$ acts on gene expression in a complex tissue that includes lymphocytes, stromal, vascular smooth muscle, endothelial and decidual cells, a cDNA microarray $\left(\right.$ GEM $^{\circledR}$, Incyte, St Louis, MO) was undertaken. Maternal mesometria from $\mathrm{C} 57 \mathrm{Bl} / 6 \mathrm{~J}$ mice at day 6 to day 10 of gestation were compared. Forty-three genes, documented among the hundreds regulated by IFN- $\gamma$, were present on the array and differentially expressed (B. A. Croy, unpublished). Among these IFN- $\gamma$-regulated genes were mouse $\alpha_{2}$-macroglobulin (MAM) and its receptor. A related EST777415, defined as $\alpha_{2}$-macroglobulin $\left(\alpha_{2} M\right)$ precursor-like, was among the most differentially upregulated genes. The array results were extended by northern blot analyses. Non-decidualized mouse uterus (virgin and at day 3 of gestation) did not express EST777415 but expression was induced by day 6 of gestation, peaked at days 10 and 12 of gestation and then declined by day 14 of gestation $(\mathrm{H}$. He, D. McCartney, Q. Wei, S. Esadeg, J. Zhang, A. Hayes, F. van Leuven and B. A. Croy, unpublished). Members of the $\alpha_{2} M$ family are abundant plasma proteins that regulate the bioavailability of proteases and cytokines in tissue (Borth, 1994). The genes map closely in mice to the NK cell LY49 receptor gene complex on chromosome 6 (Ensembl, Mouse Genome Browser BLASTView).

Potential roles of MAM and its related, expressed family member murinoglobulin-1 (MUG-1) in implantation sites were addressed by undertaking histological analyses using MAM $\% / M U G-1 \%$ mice (Umans et al., 1999). Placental development was highly unusual; the labyrinth was reduced whereas giant cells were in excess (Fig. 3). In addition, intramural trophoblast invasion of the SA was aggressive and by mid-gestation reached the myometrial circular smooth muscle layer, a position normally achieved in very late gestation. UNK cells were numerous and SA, although cuffed by trophoblast, were dilated. This indicated that members of the $\alpha_{2} M$ gene family regulate the rate at which trophoblast invades and led the authors to characterize EST777415, which remains expressed in decidua of $\mathrm{MAM}^{\circ} / \mathrm{MUG}-1^{\%}$ (Esadeg, 2002).

A full-length cDNA, now designated $\alpha_{2} \mathrm{M}$ of mouse pregnancy (A2Mp) was obtained from EST777415 (Genbank accession no. AY185125). The predicted amino acid sequence is typical of members of the $\alpha_{2} M$ family with bait, thiol ester and receptor-binding domains. The bait region is most distinctive. The predicted $\mathrm{A} 2 \mathrm{Mp}$ amino acid sequence is closest to rat $\alpha_{2} \mathrm{M}$, an acute phase protein $(90 \%)$, and $71 \%$ and $63 \%$ matched to the non-acute phase reactants human $\alpha_{2} \mathrm{M}$ and pregnancy zone protein, a molecule of undefined function, which is highly upregulated during human gestation. Homology to the four other $\alpha_{2} \mathrm{M}$ mouse family members is at $50 \%(\mathrm{H}$. He, D. McCartney, Q. Wei, S. Esadeg, J. Zhang, A. Hayes, F. van Leuven and B. A. Croy, unpublished). Unlike other members of the mouse gene family, A2Mp is not detected in liver by RT-PCR or by in situ hybridization. Rather, testes and ovaries constitutively transcribe A2Mp; uterus transcribes A2Mp only with decidualization and the gene is transcribed in lactating mammary gland. In situ hybridizations (Fig. 4) localized A2Mp to primary spermatocytes and Sertoli cells in testes and to granulosa cells of secondary and pre-ovulatory ovarian follicles, in patterns distinct from those reported for MAM (Zhu et al., 1994; Dajee et al., 1998). Expression in $24 \mathrm{~h}$ postpartum, lactating mammary tissue was localized to alveolar epithelium $(\mathrm{H}$. He, D. McCartney, Q. Wei, S. Esadeg, J. Zhang, A. Hayes, F. van Leuven and B. A. Croy, unpublished). Uterine localization was dynamic. At day 6 of gestation, A2Mp was expressed by anti-mesometrial decidua. Signal then migrated mesometrially and by days 8-14 of gestation was in vascular smooth muscle of SA and unmodified fibroblasts interfaced between mesometrial myometrium and decidua (full description of these experiments will be published elsewhere). One interpretation of these findings is that A2Mp binds molecules needed for cells 


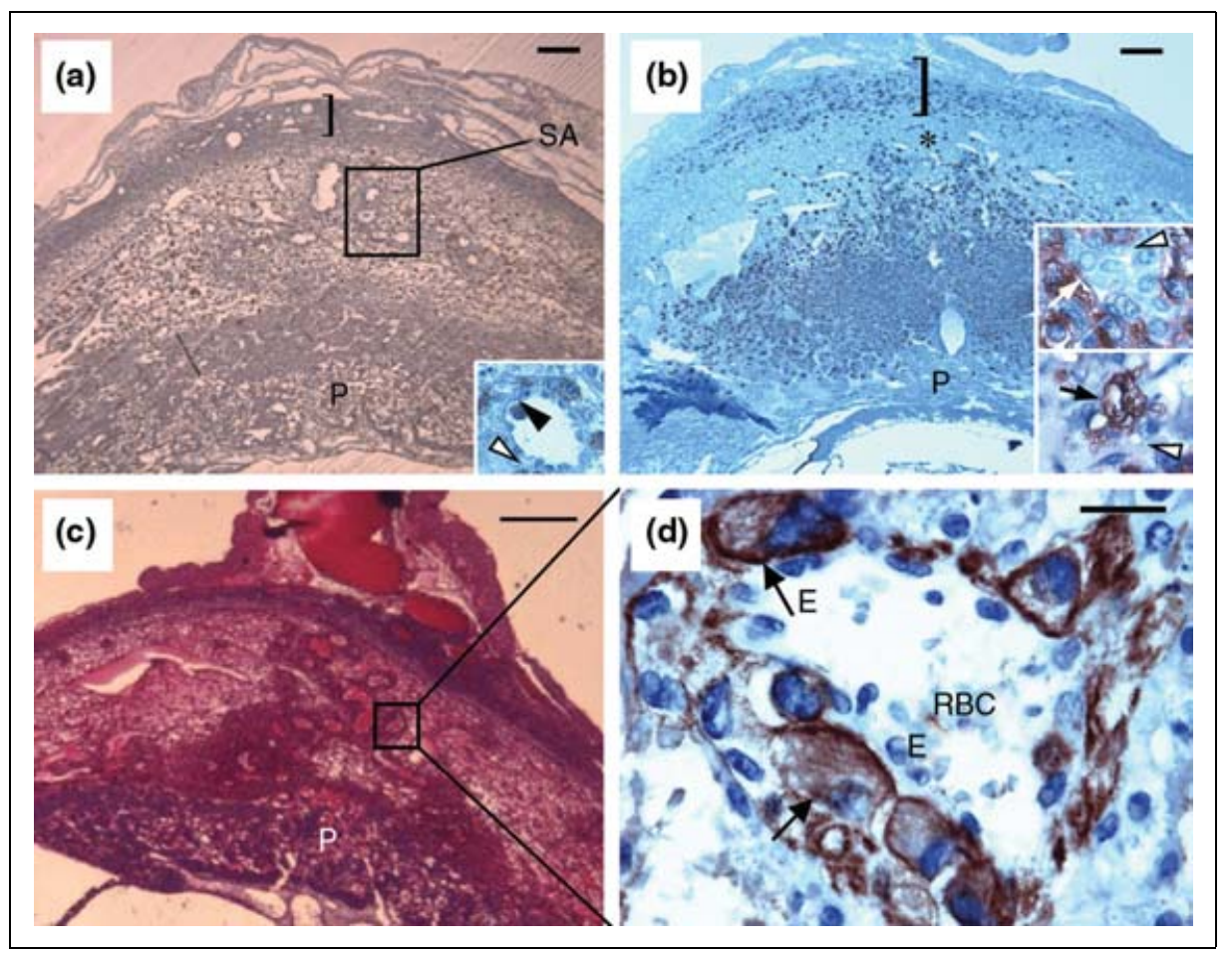

Fig. 3. Photomicrographs of implantation sites at day 10 of gestation in mice with different genetic mutations. (a) An implantation site in IL-12\%/IL-18\%. These mice have normal uterine natural killer (uNK) cell differentiation and mesometrial lymphoid aggregate of pregnancy (MLAp) (]) and placental (P) development but lower than normal levels of interferon gamma (IFN- $\gamma$ ) at midgestation and only limited modification of the spiral arteries (SA). Intravascular (black arrowhead) and perivascular (white arrowhead) localization of uNK cells is seen in the decidua basalis of these mice (insert). (b) An implantation site in IFN- $\gamma^{\circ / 0}$ with over-development of uNK cells and the MLAp (]). uNK cells are unusually localized in decidua basalis close to the placenta (P), leaving a band of decidua relatively void of uNK cells $(*)$. Upper insert shows this region at day 10 of gestation with healthy decidual cells (white arrow) clustered with darker, Dolichos biflorus agglutinin (DBA)-lectin stained uNK cells (arrowhead). Lower insert is of the same region at day 12 of gestation, when the decidua basalis has become necrotic (Ashkar et al., 2000). Decidual cells have been destroyed and amorphous material fills the spaces between cells. Small mononuclear cells and fibroblasts (black arrow) replace the decidual cell population. uNK cells (arrowhead) appear less granulated at this time point (day 12 of gestation) than in normal mice. No spiral artery modification occurs in IFN- $\gamma^{\circ / \circ}$ pregnancy. (c,d) Implantation sites in $\mathrm{MAM}^{\circ} / \mathrm{MUG}-1^{\%}$ with relative overgrowth of trophoblast particularly along the spiral artery (P: placenta); (d) is a higher magnification $(\times 1000)$ of the boxed area in (c) on a serial section stained with anti-cytokeratin antibody and illustrates intramural trophoblast cells. Erythrocytes (RBC) are present in the spiral artery lumen and endothelial cells (E) were always present between trophoblasts (arrow) and circulating cells in this segment of the vessel. Scale bars represent (a,b) $200 \mu \mathrm{m}$, (c) $400 \mu \mathrm{m}$ and (d) $20 \mu \mathrm{m}$. (a,b) Stained with DBA lectin and haematoxylin; (c) stained with haematoxylin and eosin; and (d) counter-stained with haematoxylin.

in reproductive tissues to transition from quiescence to active differentiation.

The present authors reasoned that if the A2Mp genederived product was the molecule of key importance induced by uNK cell-derived IFN- $\gamma$, administration of A2Mp to pregnant IFN- $\gamma^{\circ}$ mice would induce SA modification. As no gene product is yet available but homology is significant to human $\alpha_{2} M$, human plasmaderived $\alpha_{2} M$ or PBS was infused from days 6-11 of gestation into pregnant IFN- $\gamma^{\circ / \%}$ or alymphoid mice.
In both stains, native human $\alpha_{2} \mathrm{M}$ induced full SA modification whereas PBS induced no modification (H. He, D. McCartney, Q. Wei, S. Esadeg, J. Zhang, A. Hayes, F. van Leuven and B. A. Croy, unpublished). Thus, human $\alpha_{2} M$ must have regulated proteases, cytokines or other molecules that signal vascular dilation and elongation during gestation. When human $\alpha_{2} M$, activated by methylamine to destroy its protease binding sites, was infused into pregnant alymphoid mice, SA dilation was again achieved. This experiment indicates 


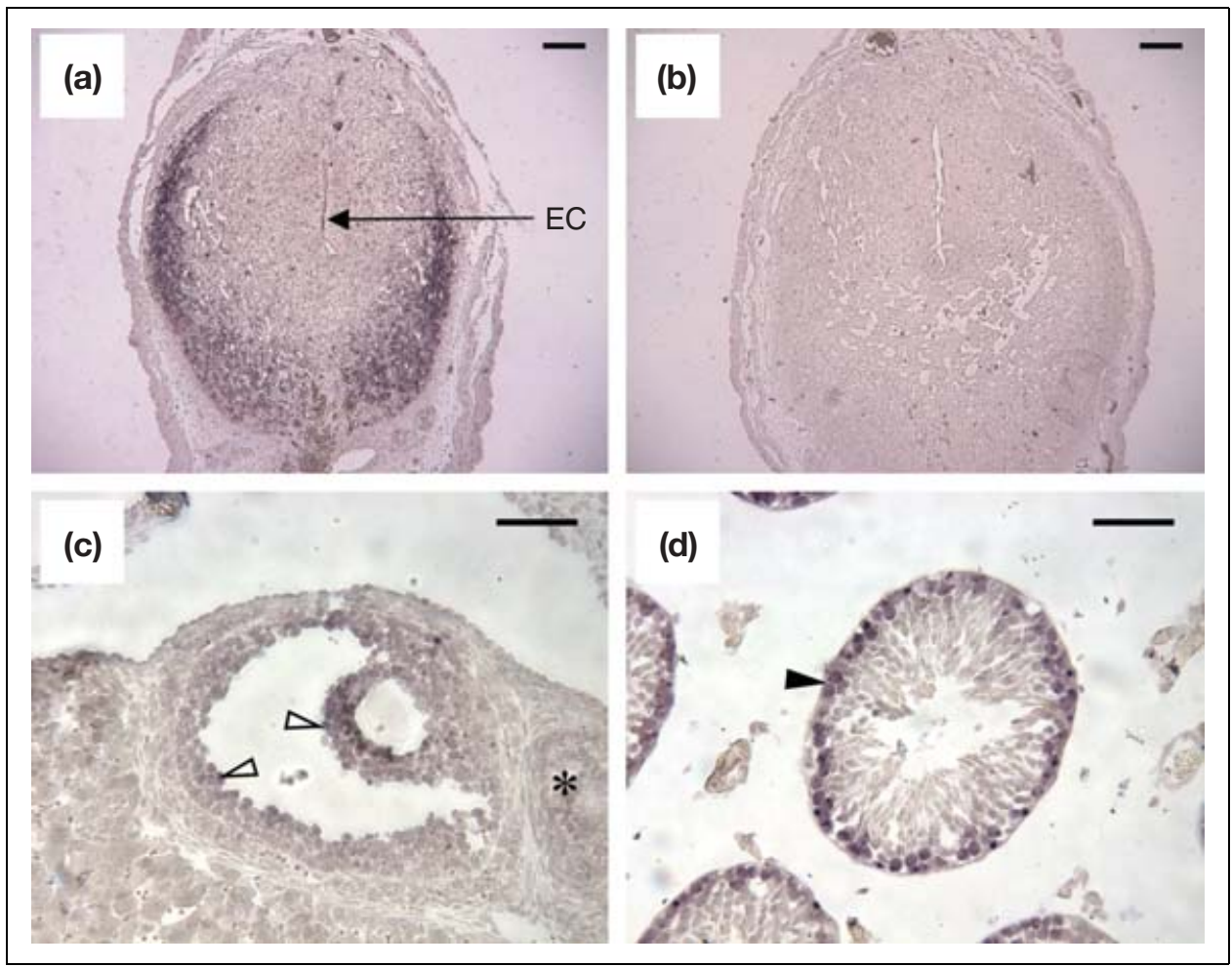

Fig. 4. In situ hybridization for $\alpha_{2} \mathrm{M}$ of mouse pregnancy (A2Mp) in reproductive tissues. (a) Strong hybridization of an anti-sense DIG-labelled probe to anti-mesometrial decidual cells at day 6 of gestation. The embryonic crypt is labelled EC. (b) The sense strand hybridization negative control. (c) A secondary follicle in the ovary, showing hybridization to granulosa cells (arrowheads). Primary follicles $(*)$ and corpora lutea (not shown) were unlabelled. (d) Localized expression of A2Mp in a seminiferous tubule from a mature $\mathrm{C} 57 \mathrm{Bl} / 6 \mathrm{~J}$ mouse (arrowhead). Hybridization appeared restricted to spermatogonia, primary spermatocytes and Sertoli cells. Scale bars represent $(\mathrm{a}, \mathrm{b}) 400 \mu \mathrm{m}$ and (c,d) $50 \mu \mathrm{m}$.

that cytokines rather than proteases are the key molecules involved in SA destabilization. If $\mathrm{A} 2 \mathrm{Mp}$ is the key molecule induced by uNK cell-derived IFN- $\gamma$ and if A2Mp, through its bound molecules, effects SA dilation and elongation, it is not necessary to postulate additional vasoactive uNK cell products. However, these are known. In mice, inducible nitric oxide synthase, the enzyme generating the powerful vasodilator $\mathrm{NO}$, is predominantly found in uNK cells of normal implantation sites (Hunt et al., 1997). In contrast, implantation sites in UNK cell-deficient mice display expression of this enzyme in trophoblast (Hunt et al., 1997). Vascular endothelial cell growth factor (VEGF), a molecule bound by $\alpha_{2} \mathrm{M}$, is produced by murine and human uNK cells (Wang et al., 2000; Li et al., 2001). In humans, placenta growth factor, a molecule upregulating the bioavailability of VEGF, is reported to be expressed exclusively in UNK cells (Li et al., 2001). Collectively these studies indicate that murine and human uNK cells contribute in various ways to the maternal vascular changes that occur in support of pregnancy.

\section{Conclusions}

Rodent studies strongly indicate that uNK cells are key contributors to maternal uterine changes induced by and in support of pregnancy. These studies indicate that many regulatory steps are involved, each of which could have a clinical correlate culminating in syndromes such as preeclampsia or intrauterine growth retardation. Although it is appealing to suggest that uNK cell-deficient mice may model pre-eclampsia, the present authors' opinion is that they model only vascular aspects. Serial urinalysis of mated NK/UNK cell-deficient IL- $15^{\circ / 0}$ and C57BI/6 mice for protein (Lowry technique) failed to show proteinurea in uNK cell-deficient mice above that seen in pregnant normal mice (Fig. 5). Other strains, such as BMP5, which shows pregnancy-induced glomerulosclerosis, proteinurea and hypertension (Davisson et al., 2002), or thrombomodulin $\%$, which displays trophoblast death and fibrinoid deposition (Isermann et al., 2003), are important and investigations of pre-eclampsia may be best served by combining these or other strains as murine 


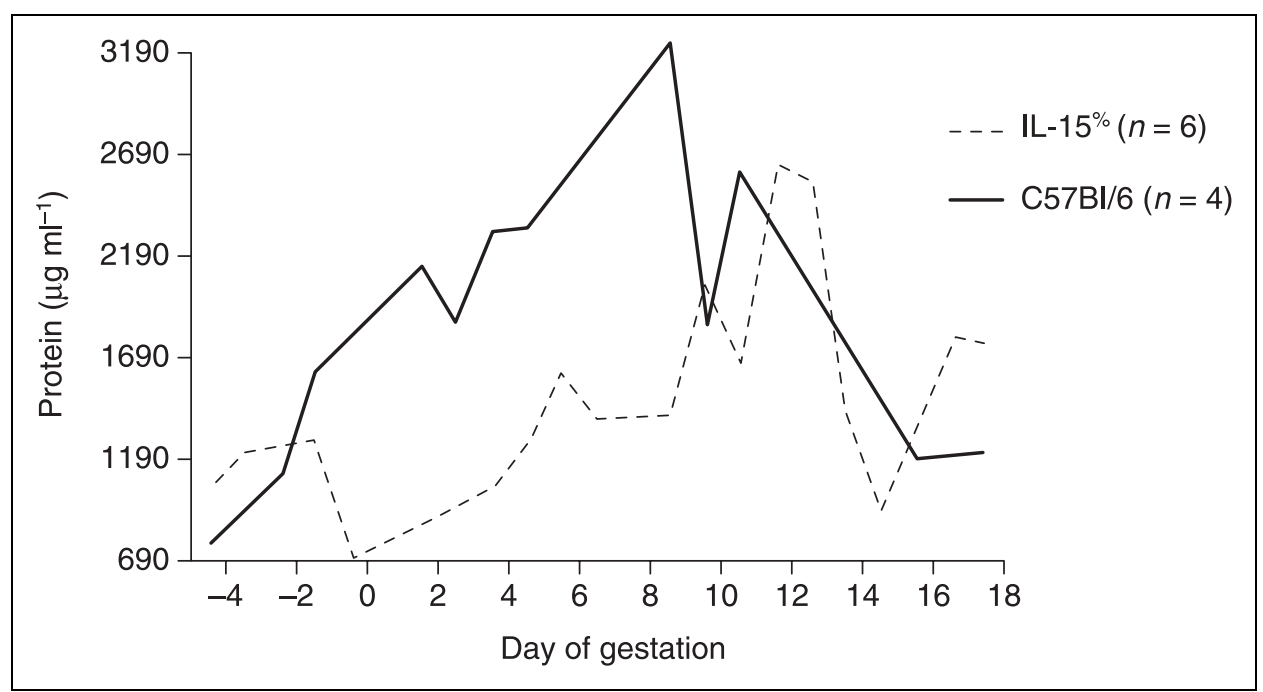

Fig. 5. Analysis of mean urinary protein (Lowry assay) collected daily from individual uterine natural killer (uNK) cell-deficient IL-15\% mice (dashed line; $n=6$ ) and C57BI/6J mice (solid line; $n=4$ ) from one oestrous cycle (-4-0) and pregnancy (0-18). Every day, individual mice were placed in clean cages having a base composed of sterile 96 -well plates. The mice were allowed to roam freely and void. Wells containing urine but no faeces were collected and pooled when urine volumes of 50-200 $\mu \mathrm{l}$ appeared to be available. The uNK cell-deficient mice were less proteinuric than their gestation-day matched normal congenic controls.

study models. Although there are many differences between implantation sites in rodents and women, this review illustrates the dynamic and detailed information that can be collected from serial time-course studies of murine pregnancy to provide valuable concepts in understanding the biology of human CD56 bright uterine cells. The resolving power of mouse genetics, combined with transplantation, in addressing questions of regulatory mechanisms in mammalian pregnancy in vivo are illustrated. The authors hope that others will develop and explore further variations of these approaches.

This study was supported by awards from the Natural Sciences and Engineering Council, Canada and the Ontario Ministry of Agriculture, Food and Rural Affairs. The authors thank the many collaborators who contributed genetically manipulated mice, advice and other assistance to the study. Immunohistology for Rae1 was conducted by T. Seya and Y. Nishizawa, University of Osaka Prefecture. The authors also thank the staff of the OMAFRA animal housing facility, Guelph, for their concerns for the health of the immune deficient mice, their dedicated care of these animals and for teaching barrier husbandry procedures to all of the trainees who have contributed to this work.

\section{References}

Key references are identified by asterisks.

Ashkar AA and Croy BA (1999) Interferon regulatory factor-1 has important functions in placental growth and modification of decidual spiral arteries Placenta 20 A17 (Abstract)

*Ashkar AA, Di Santo JP and Croy BA (2000) Interferon gamma contributes to initiation of uterine vascular modification, decidual integrity, and uterine natural killer cell maturation during normal murine pregnancy Journal of Experimental Medicine 192 259-270

Borth W (Ed) (1994) Biology of alpha 2 macroglobulin, its receptor, and related proteins Annals New York Academy of Sciences 737 1-521

Borzychowski AM, Chantakru S, Minhas K, Paffaro VA, Jr, Yamada AT, He H, Korach KS and Croy BA (2003) Functional analysis of murine uterine natural killer cells genetically devoid of estrogen receptors Placenta 24 403-411

Bulmer JN, Hollings D and Ritson A (1987) Immunocytochemical evidence that endometrial stromal granulocytes are granulated lymphocytes Journal of Pathology 153 281-288

*Campbell JJ, Qin S, Unutmaz D, Soler D, Murphy KE, Hodge MR, Wu L and Butcher EC (2001) Unique subpopulations of CD56+ NK and NK-T peripheral blood lymphocytes identified by chemokine receptor expression repertoire Journal of Immunology 166 6477-6482

Carson WE, Giri JG, Lindemann MJ, Linett ML, Ahdieh M, Paxton R, Anderson D, Eisenmann J, Grabstein K and Caligiuri MA (1994) Interleukin (IL) 15 is a novel cytokine that activates human natural killer cells via components of the IL-2 receptor Journal of Experimental Medicine 180 1395-1403

Cerwenka A and Lanier L (2001) Ligands for natural killer cell receptors: redundancy or specificity Immunology Reviews 181 158-169

Chantakru S (2002) Characterization of Migration of Natural Killer Cells to the Uterus During Pregnancy PhD Thesis, University of Guelph

*Chantakru S, Miller C, Roach LE, Kuziel WA, Maeda N, Wang WC, Evans SS and Croy BA (2002) Contributions from self-renewal and trafficking to the uterine NK cell population of early pregnancy Journal of Immunology $16822-28$

Chaouat G, Zourbas S, Ostojic S, Lappree-Delage G, Dubanchet S, Ledee $\mathbf{N}$ and Martal J (2002) A brief review of recent data on some cytokine expressions at the materno-foetal interface which might challenge the classical Th1/Th2 dichotomy Journal of Reproductive Immunology 53 241-256

Chegini N, Ma C, Roberts M, Williams RS and Ripps BA (2002) Differential expression of interleukins (IL) IL-13 and IL-15 throughout the menstrual cycle in endometrium of normal fertile women and women with recurrent spontaneous abortion Journal of Reproductive Immunology 56 93-110 
Christmas SE, Bulmer JN, Meager A and Johnson PM (1990) Phenotypic and functional analysis of human CD3- decidual leucocyte clones Immunology 71 182-189

Couse JF and Korach KS (1999) Estrogen receptor null mice: what have we learned and where will they lead us? Endocrinology Reviews 20 358-417

Croy BA, Esadeg S, Chantakru S, van den Heuvel M, Paffaro VA, Jr, He H, Black GP, Ashkar AA, Kiso Y and Zhang J (2003) Update on pathways regulating the activation of uterine natural killer cells, their interactions with decidual spiral arteries and homing of their precursors to the uterus Journal of Reproductive Immunology 57 151-168

Dajee M, Georg HF and Richards JS (1998) Stat 5b and the orphan nuclear receptors regulate expression of the $\alpha_{2}$-macroglobulin gene in rat ovarian granulosa cells Molecular Endocrinology 12 1393-1409

Davisson RL, Hoffmann DS, Butz GM, Aldape G, Schlager G, Merrill DC, Sethi S, Weiss RM and Bates JN (2002) Discovery of a spontaneous genetic mouse model of preeclampsia Hypertension 39 337-342

Delgado SR, McBey BA, Yamashiro S, Fujita J, Kiso Y and Croy BA (1996) Accounting for the peripartum loss of granulated metrial gland cells, a natural killer cell population, from the pregnant mouse uterus Journal of Leukocyte Biology 59 262-269

*Devergne O, Coulomb-L'Hermine A, Capel F, Moussa M and Capron F (2001) Expression of Epstein-Barr virus-induced gene 3, an interleukin12 p40-related molecule, throughout human pregnancy: involvement of syncytiotrophoblasts and extravillous trophoblasts American Journal of Pathology 159 1763-1776

Dunn CL, Critchley HO and Kelly RW (2002) IL-15 regulation in human endometrial stromal cells Journal of Clinical Endocrinology and Metabolism 87 1898-1901

Esadeg SM (2002) Studies on the Alpha 2 Macroglobulkin Gene Family in Pregnant Mouse Uterus MSc Thesis, University of Guelph

Evans SS, Wang WC, Bain MD, Burd R, Ostberg JR and Repasky EA (2001) Fever-range hyperthermia dynamically regulates lymphocyte delivery to high endothelial venules Blood 97 2727-2733

Fu YX and Chaplin DD (1999) Development and maturation of secondary lymphoid tissues Annual Reviews of Immunology 17 399-433

Greenwood JD, Minhas K, Di Santo JP, Makita M, Kiso Y and Croy BA (2000) Ultrastructural studies of implantation sites from mice deficient in uterine natural killer cells Placenta 21 693-702

Guimond MJ, Wang B and Croy BA (1998) Engraftment of bone marrow from severe combined immunodeficient (SCID) mice reverses the reproductive deficits in natural killer cell-deficient tg epsilon 26 mice Journal of Experimental Medicine 187 217-223

Henderson TA, Saunders PT, Moffett-King A, Groome NP and Critchley HO (2003) Steroid receptor expression in uterine natural killer cells Journal of Clinical Endocrinology and Metabolism 88 440-449

Hiby SE, King A, Sharkey AM and Loke YW (1997) Human uterine NK cells have a similar repertoire of killer inhibitory and activatory receptors to those found in blood, as demonstrated by RT-PCR and sequencing Molecular Immunology 34 419-443

Hunt JS, Miller L, Vassmer D and Croy BA (1997) Expression of the inducible nitric oxide synthase gene in mouse uterine leukocytes and potential relationships with uterine function during pregnancy Biology of Reproduction $\mathbf{5 7}$ 827-836

Isermann B, Sood R, Pawlinski R, Zogg M, Kalloway S, Degen JL, Mackman N and Weiler H (2003) The thrombomodulin-protein C system is essential for the maintenance of pregnancy Nature Medicine 9 331337

Jabbour HN, Critchley HO, Yu-Lee LY and Boddy SC (1999) Localization of interferon regulatory factor-1 (IRF-1) in nonpregnant human endometrium: expression of IRF-1 is up-regulated by prolactin during the secretory phase of the menstrual cycle Journal of Clinical Endocrinology and Metabolism 84 4260-4265

Kather A, Chantakru S, He H, Minhas K, Foster R, Markert UR, Pfeffer K and Croy BA (2003) Neither lymphotoxin alpha nor lymphotoxin beta receptor expression is required for biogenesis of lymphoid aggregates or differentiation of natural killer cells in the pregnant mouse uterus Immunology 108 1-8
Kennedy MK, Glaccum M, Brown SN et al. (2000) Reversible defects in natural killer and memory CD8 T cell lineages in interleukin 15-deficient mice Journal of Experimental Medicine 191 771-780

King A (2000) Uterine leukocytes and decidualization Human Reproduction Update 6 28-36

Kitaya K, Yasuda J, Yagi I, Tada Y, Fushiki S and Honjo H (2000) IL-15 expression at human endometrium and decidua Biology of Reproduction 63 683-687

Kitaya K, Yasuda J, Fushiki S and Honjo H (2001) Localization of interferon regulatory factor- 1 in human endometrium throughout the menstrual cycle Fertility and Sterility 75 992-996

*Li XF, Charnock-Jones DS, Zhang E et al. (2001) Angiogenic growth factor messenger ribonucleic acids in uterine natural killer cells Journal of Clinical Endocrinology and Metabolism 86 1823-1834

Moffett-King A (2002) Natural killer cells and pregnancy Nature Reviews Immunology 2 656-663

Nakanishi K, Yoshimoto T, Tsutsui H and Okamura H (2001) Interleukin-18 regulates both Th1 and Th2 responses Annual Reviews of Immunology $19423-474$

Natarajan K, Dimasi N, Wang J, Mariuzza RA and Margulies DH (2002) Structure and function of natural killer cell receptors: multiple molecular solutions to self, non-self discrimination Annual Review of Immunology 20 853-885

Nomura M, Zou Z, Joh T, Takihara $Y$, Matsuda $Y$ and Shimada K (1996) Genomic structures and characterization of Rae1 family members encoding GPI-anchored cell surface proteins and expressed predominantly in embryonic mouse brain Journal of Biochemistry (Tokyo) 120 987-995

Okada S, Okada H, Sanezumi M, Nakajima T, Yasuda K and Kanzaki H (2000) Expression of interleukin-15 in human endometrium and decidua Molecular Human Reproduction 6 75-80

Paffaro VA, Jr, Bizinotto MC, Joazeiro PP and Yamada AT (2003) Subset classification of mouse uterine natural killer cells by DBA lectin reactivity Placenta 24 479-488

Parham C, Chirica M, Timans J et al. (2002) A receptor for the heterodimeric cytokine IL-23 is composed of IL-12Rbeta1 and a novel cytokine receptor subunit, IL-23R Journal of Immunology 168 5699-5708

Parr EL, Parr MB and Young JD (1987) Localization of a pore-forming protein (perforin) in granulated metrial gland cells Biology of Reproduction $\mathbf{3 7}$ 1327-1335

Peel S (1989) Granulated metrial gland cells Advances in Anatomy Embryology and Cell Biology 115 1-112

Pflanz S, Timans JC, Cheung J et al. (2002) IL-27, a heterodimeric cytokine composed of EBI3 and p28 protein, induces proliferation of naive $\mathrm{CD} 4(+)$ T cells Immunity 16 779-790

Rosmaraki EE, Douagi I, Roth C, Colucci F, Cumano A and Di Santo JP (2001) Identification of committed NK cell progenitors in adult murine bone marrow European Journal of Immunology 31 19001909

Searle RF, Jones RK and Bulmer JN (1999) Phenotypic analysis and proliferative responses of human endometrial granulated lymphocytes during the menstrual cycle Biology of Reproduction 60 871878

Takeda K, Tsutsui H, Yoshimoto T, Adachi O, Yoshida N, Kishimoto T, Okamura H, Nakanishi K and Akira S (1998) Defective NK cell activity and Th1 response in IL-18-deficient mice Immunity 8 383-390

Trinchieri G (1995) Interleukin-12: a proinflammatory cytokine with immuno-regulatory functions that bridge innate resistance and antigenspecific adaptive immunity Annual Reviews of Immunology 13251 276

Umans L, Serneels L, Overbergh L, Stas L and Van Leuven F (1999) alpha2-macroglobulin- and murinoglobulin-1- deficient mice. A mouse model for acute pancreatitis American Journal of Pathology 155983 993

Wang C, Umesaki N, Nakamura H, Tanaka T, Nakatani K, Sakaguchi I, Ogita $\mathbf{S}$ and Kaneda K (2000) Expression of vascular endothelial growth factor by granulated metrial gland cells in pregnant murine uteri Cell and Tissue Research 300 285-293 
Ye W, Zheng LM, Young JD and Liu CC (1996) The involvement of interleukin (IL)-15 in regulating the differentiation of granulated metrial gland cells in mouse pregnant uterus Journal of Experimental Medicine 1842405 2410

Yoshino O, Osuga Y, Koga K et al. (2001) Evidence for the expression of interleukin (IL)-18, IL-18 receptor and IL-18 binding protein in the human endometrium Molecular Human Reproduction 7649 654

Zhang JH, He H, Borzychowski AM, Takeda K, Akira S and Croy BA (2003) Analysis of cytokine regulators inducing interferon-gamma pro- duction by mouse uterine natural killer cells Biology of Reproduction DOI: 10.1095/biolreprod.103.015529

Zhu LJ, Cheng CY, Phillips DM and Bardin CW (1994) The immunohistochemical localization of alpha 2-macroglobulin in rat testes is consistent with its role in germ cell movement and spermiation Journal of Andrology 15 575-582

Zourbas S, Dubanchet S, Martal J and Chaouat G (2001) Localization of pro-inflammatory (IL-12, IL-15) and anti-inflammatory (IL-11, IL13) cytokines at the foeto-maternal interface during murine pregnancy Clinical and Experimental Immunology 126 519-528 